\title{
EXPERIMENTO DIDÁTICO DE QUIMIOMETRIA PARA CALIBRAÇÃO MULTIVARIADA NA DETERMINAÇÃO DE PARACETAMOL EM COMPRIMIDOS COMERCIAIS UTILIZANDO ESPECTROSCOPIA NO INFRAVERMELHO PRÓXIMO: UM TUTORIAL, PARTE II
}

\author{
André Marcelo de Souza\#, Márcia Cristina Breitkreitz, Paulo Roberto Filgueiras, Jarbas José Rodrigues Rohwedder e \\ Ronei Jesus Poppi* \\ Departamento de Química Analítica, Instituto de Química, Universidade Estadual de Campinas, CP 6154, 13084-971 Campinas \\ - SP, Brasil
}

Recebido em 10/10/12; aceito em 6/2/13; publicado na web em 15/4/13

\begin{abstract}
TEACHING EXPERIMENT OF CHEMOMETRICS FOR MULTIVARIATE CALIBRATION IN DETERMINATION OF PARACETAMOL IN COMMERCIAL TABLETS USING NEAR-INFRARED SPECTROSCOPY: A TUTORIAL, PART II. The aim of this manuscript was to show the basic concepts and practical application of Partial Least Squares (PLS) as a tutorial, using the Matlab computing environment for beginners, undergraduate and graduate students. As a practical example, the determination of the drug paracetamol in commercial tablets using Near-Infrared (NIR) spectroscopy and Partial Least Squares (PLS) regression was shown, an experiment that has been successfully carried out at the Chemical Institute of Campinas State University for chemistry undergraduate course students to introduce the basic concepts of multivariate calibration in a practical way.
\end{abstract}

Keywords: NIR; multivariate calibration; teaching experiment.

\section{INTRODUÇÃO}

Este trabalho é uma continuação da série de tutoriais que tem como objetivo a elaboração de experimentos didáticos de Quimiometria a serem utilizados como um guia prático em cursos de graduação e pós-graduação em química e áreas afins. No primeiro tutorial, ${ }^{1}$ a Análise por Componentes Principais (Principal Component Analysis, PCA) foi abordada em um experimento de análise exploratória de amostras de óleos vegetais comestíveis analisados por espectroscopia no infravermelho médio com transformada de Fourier. Neste primeiro trabalho, algumas das operações básicas e fundamentais para a execução da PCA em Matlab foram apresentadas com o objetivo de possibilitar ao iniciante em Quimiometria, mesmo que em um primeiro contato com este programa computacional, carregar os dados, montar as matrizes, criar e formatar os gráficos, além de ter uma visão mais prática da PCA. Este tipo de abordagem não é comum na literatura especializada, além de não existirem livros textos em português voltados para este tema.

Neste segundo trabalho, será abordado outro tema de fundamental importância em Quimiometria: a calibração multivariada. Para essa finalidade, um dos métodos de calibração mais utilizados, a Regressão por Quadrados Mínimos Parciais (Partial Least Squares, PLS) foi selecionado para o tratamento dos dados. Como no trabalho anterior, o PLS foi executado em ambiente computacional Matlab, devido principalmente à elevada aceitação e versatilidade deste programa, permitindo que o usuário crie e adapte suas próprias rotinas. Apesar da grande versatilidade do Matlab, existe uma dificuldade por parte dos iniciantes em utilizá-lo e, por este motivo, é interessante que os comandos básicos e específicos para as aplicações quimiométricas sejam apresentados. Um diferencial deste segundo trabalho, em relação ao primeiro, é o aprofundamento na utilização do Matlab para fins quimiométricos, em que novas

*e-mail: ronei@iqm.unicamp.br

\# Endereço atual: Embrapa Solos, Empresa Brasileira de Pesquisa Agropecuária - Rua Jardim Botânico, 1.024 - Jardim Botânico - CEP 22460-000 - Rio de Janeiro- RJ - Brasil. funções e a capacidade de criar pequenas rotinas são apresentadas aos iniciantes.

Os dados provenientes da espectroscopia no infravermelho próximo (NIR, Near Infrared) foram selecionados para determinar a porcentagem de paracetamol em amostras de medicamentos comerciais. A espectroscopia no infravermelho próximo é um tipo de espectroscopia vibracional ${ }^{2}$ que emprega radiação eletromagnética na faixa de energia de $2,65 \times 10^{-19}$ a 7,96 x $10^{-20} \mathrm{~J}$, a qual corresponde à faixa de comprimentos de onda de 750 a $2.500 \mathrm{~nm}$ (ou números de onda de 13.300 a $4.000 \mathrm{~cm}^{-1}$ ). Nesta região são detectadas as vibrações de primeiro, segundo e terceiro sobretons (overtones) de bandas fundamentais observadas no infravermelho médio bem como as bandas de combinação. Sobretons são transições múltiplas das transições fundamentais, ou seja, acontecem do nível vibracional fundamental $n=0$ para os diversos níveis vibracionais $n=2,3,4 \ldots$ A regra de seleção para o infravermelho (considerando a molécula como um oscilador harmônico) é $\Delta \mathrm{n}= \pm 1$ e, desta maneira, as transições de sobretons seriam consideradas proibidas. No entanto, sobretons acontecem principalmente devido ao fenômeno de anarmonicidade mecânica, observado para muitas moléculas que apresentam diferenças entre as massas dos átomos conectados pela ligação química. Nestes casos, a vibração não pode ser descrita por um oscilador com movimento harmônico. As bandas de combinação são obtidas a partir da combinação (pela soma ou diferença dos números de onda) para fornecer uma única banda e sua presença no espectro NIR indica que as vibrações não são independentes. A sobreposição de muitos sobretons e bandas de combinação leva à falta de especificidade espectral na região NIR, ao contrário da região do infravermelho médio, onde picos específicos podem ser atribuídos a diferentes grupos químicos.

Para que ocorra absorção da radiação NIR, a energia do fóton incidente deve ser idêntica à diferença de energia entre dois níveis vibracionais. Entretanto, este critério não é suficiente e, para uma vibração ser ativa, é também necessário que o campo elétrico oscilante da radiação incidente possa interagir com a molécula. Isto só pode acontecer se o deslocamento dos átomos na vibração produzir uma mudança no momento de dipolo da molécula. A intensidade da absorção da radiação NIR por um sistema é proveniente da variação 
do momento de dipolo sendo a frequência da radiação absorvida idêntica à da oscilação do dipolo. Por apresentarem alta anarmonicidade e grande momento de dipolo, as principais bandas de absorção na região do infravermelho próximo são relacionadas à sobretons e bandas de combinação das transições vibracionais fundamentais dos grupos: C-H, N-H, O-H e S-H. Um aprofundamento sobre a teoria, instrumentação e atribuição de bandas para a espectroscopia NIR pode ser encontrado em livros ${ }^{3,4}$ e artigos científicos. ${ }^{5,6}$ Uma edição especial dedicada à espectroscopia NIR foi publicada em $2005 \mathrm{e}$ contém aspectos históricos, instrumentação e aplicações nas mais diversas áreas. ${ }^{7}$

A espectroscopia NIR e a Quimiometria convivem em simbiose. Uma vez que o espectro NIR possui bandas largas e sobrepostas, a Quimiometria torna-se uma ferramenta valiosa para retirar as informações contidas nos espectros NIR permitindo a identificação e a quantificação de diversos parâmetros em diferentes matrizes. Além disso, a própria Quimiometria se beneficia do tipo e da elevada quantidade de informação espectral gerada para o desenvolvimento de novos métodos empregando espectroscopia NIR. Na realidade, a quantificação de parâmetros analíticos empregando espectroscopia NIR sem a separação prévia do analito da matriz, na maioria dos casos apenas é possível empregando métodos de calibração multivariada, uma vez que dificilmente um sinal específico (livre de interferência da matriz) é observado para o analito na região do NIR. A possibilidade de quantificar o analito sem a necessidade de separá-lo da matriz é uma grande vantagem da espectroscopia NIR em relação às técnicas de separação, como a cromatografia líquida de alta eficiência, uma vez que o desenvolvimento de métodos analíticos exige pouco ou nenhum tratamento da amostra, minimizando a geração de resíduos. Além disso, o tempo de preparo da amostra normalmente é muito inferior em relação aos métodos cromatográficos e, consequentemente, o custo das análises também é reduzido. O limite de detecção da técnica permite a análise de componentes que estejam em concentrações aproximadamente superiores a $0,5 \% \mathrm{~m} / \mathrm{m}$. Considerando que a faixa de concentração de fármacos em medicamentos é normalmente superior ao limite mencionado, a espectroscopia NIR associada a métodos de calibração multivariada é uma opção atraente e limpa (de acordo com os princípios da química verde) para a realização de análises na indústria farmacêutica.

A PLS é um método de calibração multivariada que utiliza a técnica de análise de componentes principais para a redução da dimensionalidade do conjunto de dados para posterior correlação entre os espectros (matriz $\mathbf{X}$ ) e as propriedades de interesse (matriz Y). Quando existe apenas uma propriedade, $\mathbf{y}$ é um vetor e, neste caso, o método é denominado PLS 1. A propriedade de interesse muitas vezes é a concentração de um analito, porém não limitada a esta, podendo até mesmo ser propriedades físico-químicas, tais como densidade e viscosidade, as quais são relacionadas a composição da amostra. As matrizes $\mathbf{X}$ e $\mathbf{Y}$ são decompostas por PCA por meio das Equações 1 e $2 .{ }^{8}$

$$
\begin{aligned}
& \mathbf{X}=\mathbf{T} \mathbf{P}^{\mathrm{T}}+\mathbf{E} \\
& \mathbf{Y}=\mathbf{U} \mathbf{Q}^{\mathbf{T}}+\mathbf{F}
\end{aligned}
$$

sendo: $\mathbf{P}$ e $\mathbf{Q}$ são os pesos de $\mathbf{X}$ e $\mathbf{Y}$, respectivamente; $\mathbf{T}$ e $\mathbf{U}$ são os escores de $\mathbf{X}$ e $\mathbf{Y}$, respectivamente e $\mathbf{E}$ e $\mathbf{F}$ representam as matrizes de resíduos de $\mathbf{X}$ e $\mathbf{Y}$, respectivamente.

Os escores e pesos representam, respectivamente, as coordenadas das amostras no sistema de componentes principais e a contribuição relativa de cada variável original (comprimento de onda) para a formação dos mesmos. No PLS, os valores dos escores nas matrizes $\mathbf{T}$ e $\mathbf{U}$ são alterados até que o melhor modelo linear seja estabelecido entre eles e assim, a maior covariância entre $\mathbf{X}$ e $\mathbf{Y}$ seja alcançada. Isso é feito em uma série de etapas, que são repetidas para cada componente principal estimada por meio de um processo iterativo. Desta maneira, no PLS existe um compromisso entre a explicação da variância em $\mathbf{X}$ e a obtenção da maior correlação com Y. Uma vez que no cálculo de cada componente principal os valores de concentração dos analitos também são levados em consideração, as componentes principais passam a ser denominadas variáveis latentes. Quando existe mais de uma coluna na matriz e a regressão é realizada empregando todas as colunas simultaneamente, o método passa a ser denominado PLS 2. Este método é interessante em situações nas quais as propriedades de interesse estão correlacionadas. Caso contrário, é preferível realizar uma regressão empregando PLS 1 para cada coluna da matriz Y, gerando um modelo ótimo para a previsão de cada analito.

Muitas vezes é necessário realizar uma etapa de pré-processamento dos espectros para remover informações físicas e/ou instrumentais que não estão relacionadas à informação química de interesse da amostra. Alguns dos métodos de pré-processamento foram apresentados no primeiro tutorial ${ }^{1}$ e uma revisão foi realizada por Rinnan et al. ${ }^{9}$ Neste tutorial, o método de correção de espalhamento multiplicativo (MSC) foi aplicado ao conjunto de espectros com o intuito de remover flutuações aditivas e multiplicativas relacionadas à parâmetros físicos das amostras, como por exemplo, diferenças de tamanho de partícula. $\mathrm{O}$ uso de derivadas também é recomendado para esta finalidade e pode ser utilizado pelo usuário. No entanto, para o conjunto de dados descrito neste tutorial, o método MSC mostrou-se mais adequado para remover tais flutuações e garantir que os espectros contivessem apenas informações químicas relacionadas à propriedade de interesse.

O número de variáveis latentes a ser utilizado no modelo é normalmente determinado pelo procedimento de validação cruzada com o método denominado leave-one-out ("um por vez"). Neste procedimento, uma amostra (espectro) pertencente ao conjunto de calibração é deixada de fora, o modelo é construído com as demais e é realizada a previsão da propriedade de interesse para esta amostra empregando um número crescente de variáveis latentes. Este procedimento é então repetido para todas as amostras e um erro médio (RMSECV, Equação 3) é calculado para cada número de variáveis latentes empregado. O número de variáveis latentes a partir do qual não existe variação apreciável no valor de RMSECV é utilizado para determinar o número de variáveis latentes a ser utilizado no modelo. Caso exista dúvida se dois valores de RMSECV são estatisticamente diferentes, é possível a realização de um teste $\mathrm{F}$ entre os dois valores, empregando um número de graus de liberdade igual número de amostras do conjunto de calibração.

$$
R M S E C V=\sqrt{\frac{\sum\left(y_{p}-y_{e}\right)^{2}}{n}}
$$

na qual $y_{p}$ representa o valor previsto de concentração, $y_{e} o$ valor esperado e n o número de amostras de calibração.

Após a seleção do número de variáveis latentes, o modelo de regressão pode ser construído e deve ser validado empregando amostras que não participaram da calibração (validação externa). Estas amostras devem ter seus valores da propriedade de interesse previamente conhecidos, para avaliar a real capacidade de previsão do modelo. Nesta etapa, um erro quadrático médio de validação (RMSEV) é calculado, de maneira similar à Equação 3. Os valores previstos para essas amostras devem ser concordantes com os valores reais e, portanto, em um gráfico contendo estas duas grandezas, os valores das amostras devem estar próximos a uma reta com inclinação de 
$45^{\circ}$, intercepto próximo a zero (bias) e inclinação próxima à unidade. $\mathrm{O}$ coeficiente de determinação, $\mathrm{R}^{2}$, também pode ser utilizado para avaliação do ajuste. Neste trabalho também foi calculado o RMSEP (Root Mean Square Error of Prediction), referente às amostras de medicamentos comerciais, empregando os valores declarados por diferentes fabricantes para o teor de paracetamol. Muitas vezes é calculado também o RMSEC (Root Mean Square Error of Calibration), sendo que neste caso os valores previstos se referem às próprias amostras de calibração e o denominador " $n$ " deve ser subtraído do número de variáveis latentes e de uma unidade, caso os dados tenham sido centrados na média.

Neste artigo não será realizada uma discussão mais aprofundada sobre aspectos de modelagem e validação empregando PLS uma vez que serão priorizados aspectos práticos de execução do experimento. Diversos livros-texto ${ }^{10,11}$ e artigos científicos ${ }^{12,13}$ estão disponíveis na literatura para uma abordagem mais completa sobre PLS, incluindo um artigo descrevendo e comparando nove dos algoritmos mais empregados para os cálculos da regressão por PLS. ${ }^{14}$ Maiores informações sobre o desenvolvimento e validação de métodos espectroscópicos empregando calibração multivariada podem ser encontrados na norma ASTM $1655 .{ }^{15} \mathrm{Na}$ área farmacêutica, Roggo et al ${ }^{16}$ revisaram os métodos quimiométricos utilizados em diferentes aplicações empregando espectroscopia NIR e De Bleye et al ${ }^{17}$ revisaram de forma crítica alguns dos procedimentos mais aplicados para a validação de métodos empregado espectroscopia NIR.

$\mathrm{Na}$ área de educação, Yappert ${ }^{18}$ reportou a experiência de ensino de espectroscopia NIR aos cursos de graduação com um experimento para a determinação da octanagem da gasolina no qual os alunos verificaram os benefícios da Quimiometria aplicada aos espectros de bandas largas no NIR, permitindo a quantificação do parâmetro de interesse de forma rápida em uma amostra complexa como a gasolina. Ferreira et al. ${ }^{19}$ apresentaram um tutorial de calibração multivariada no qual os conceitos básicos de análise exploratória por PCA e calibração multivariada por Regressão em Componentes Principais (Principal Component Regression, PCR) e PLS foram abordados, bem como aspectos relacionados à calibração e validação dos modelos e alguns dos comandos mais importantes para a execução destes métodos quimiométricos em Matlab foram apresentados utilizando como exemplo os espectros de misturas ternárias de 2,4,6-trinitrofenolato, 2,4-dinitrofenolato e 2,5-dinitrofenolato.

Este segundo tutorial tem por objetivo geral introduzir uma proposta de experimento didático empregando Espectroscopia NIR e calibração multivariada por PLS para a determinação de paracetamol em comprimidos comerciais, utilizando o software Matlab para a realização dos cálculos. Em nosso laboratório foi simulada uma formulação de medicamento comercial de paracetamol e as amostras dos conjuntos de calibração e validação foram preparadas por alunos de graduação da disciplina de Química Analítica Instrumental. A seguir foram adquiridos medicamentos comerciais de quatro fabricantes diferentes em farmácias locais para a previsão da concentração de paracetamol empregando os espectros NIR e o modelo PLS obtido pelos alunos.

\section{PARTE EXPERIMENTAL}

\section{Materiais e reagentes}

Para a execução do experimento foi utilizado o princípio ativo Paracetamol (Synth) e os excipientes: ácido esteárico (Saiya); amido (Synth); Hidroxipropilmetil celulose (HPMC) (Pharma Nostra); polietilenoglicol (PEG) (Synth) e povidona (Vetec). Foram utilizadas quatro marcas de medicamentos comercias contendo $750 \mathrm{mg}$ de paracetamol, na forma de comprimido sem revestimento, disponíveis em Farmácias e Drogarias da região de Campinas.

\section{Preparo das amostras}

\section{Placebo}

Em papel manteiga foram pesadas as seguintes massas dos excipientes: 1,000 g ácido esteárico; 2,500 g amido; 2,000 g HPMC; 2,000g PEG e 2,500 g de povidona. Os excipientes foram transferidos, um a um, para o almofariz e homogeneizados com o auxílio de um pistilo. A mistura foi transferida para um frasco de vidro de $20 \mathrm{~mL}$ com a ajuda de um funil analítico. Em seguida, o frasco foi agitado em um vórtex por 10 minutos até a obtenção de uma mistura homogênea.

\section{Amostras de calibração}

Foram pesadas em balança analítica oitos diferentes massas de paracetamol contendo 1,$200 ; 1,300 ; 1,400 ; 1,500 ; 1,600 ; 1,700 ; 1,800$ e 1,900 g anotando as massas com precisão de mg. Cada massa de paracetamol foi adicionada a um frasco de vidro de $20 \mathrm{~mL}$ devidamente identificado. A cada um desses frascos foi adicionada uma massa de placebo necessária para que a massa final da mistura fosse igual a 2,000 g (a massa final também deve ser anotada com precisão de $\mathrm{mg}$ ). A mistura resultante foi levada ao vórtex para homogeneização por 5 minutos. Calculou-se a porcentagem de paracetamol em relação à massa total em cada amostra. A Tabela 1 apresenta um exemplo preparado por um grupo de alunos com os valores pesados de paracetamol, placebo, a massa total e a porcentagem de paracetamol na mistura final.

Tabela 1. Massas dos constituintes utilizadas no preparo das amostras de calibração e validação, porcentagem de paracetamol $(\% \mathrm{~m} / \mathrm{m})$ nestas amostras e médias calculadas para os medicamentos de cada um dos fabricantes

\begin{tabular}{ccccc}
\hline Amostra & $\begin{array}{c}\text { Paracetamol } \\
(\mathrm{g})\end{array}$ & $\begin{array}{c}\text { Placebo } \\
(\mathrm{g})\end{array}$ & $\begin{array}{c}\text { Total } \\
(\mathrm{g})\end{array}$ & $\begin{array}{c}\text { \% Paracetamol } \\
(\mathrm{m} / \mathrm{m})\end{array}$ \\
\hline C1 & 1,200 & 0,806 & 2,006 & 59,820 \\
$\mathrm{C} 2$ & 1,300 & 0,705 & 2,006 & 64,840 \\
C3 & 1,401 & 0,601 & 2,002 & 70,000 \\
C4 & 1,501 & 0,499 & 2,001 & 75,040 \\
C5 & 1,602 & 0,399 & 2,001 & 80,040 \\
C6 & 1,706 & 0,303 & 2,009 & 84,900 \\
C7 & 1,801 & 0,214 & 2,015 & 89,400 \\
C8 & 1,900 & 0,111 & 2,011 & 94,490 \\
V1 & 1,262 & 0,739 & 2,000 & 63,070 \\
V2 & 1,371 & 0,629 & 2,000 & 68,560 \\
V3 & 1,464 & 0,539 & 2,002 & 73,100 \\
V4 & 1,667 & 0,332 & 1,999 & 83,410 \\
V5 & 1,763 & 0,240 & 2,002 & 88,040 \\
Fabricante 1 & - & - & - & 89,830 \\
Fabricante 2 & - & - & - & 88,670 \\
Fabricante 3 & - & - & - & 90,910 \\
Fabricante 4 & - & - & - & 90,420 \\
\hline
\end{tabular}

\section{Amostras de validação}

Foram pesadas as seguintes massas de paracetamol e transferidas para cinco diferentes frascos previamente identificados: 1,260; 1,360; 1,$460 ; 1,660$ e $1,760 \mathrm{~g}$ anotando as massa com precisão de mg. As massas das amostras de validação foram definidas para fornecer uma porcentagem de paracetamol dentro da faixa da calibração. O procedimento adotado foi então idêntico ao realizado para as amostras de calibração: foi calculada a massa de placebo necessária 
para que a massa final da mistura fosse igual a 2,000 $\mathrm{g}$ e em seguida adiciona-se a respectiva massa de placebo. A mistura resultante foi levada ao vórtex para homogeneização por 5 minutos e foi calculada a porcentagem de paracetamol em relação à massa total em cada amostra. Os valores pesados de paracetamol, placebo, a massa total e a porcentagem de paracetamol na mistura final para a construção do modelo de validação, misturas identificadas com $\mathrm{V}$ na Tabela 1 também são mostrados como exemplo.

\section{Medicamentos comerciais}

Foram pesados dois comprimidos comerciais inteiros contendo paracetamol de quatro fabricantes diferentes e calculada a massa média dos comprimidos. Em seguida, estes comprimidos foram triturados em almofariz e transferidos para frascos previamente identificados com a marca comercial de cada um. Foi calculada a porcentagem de paracetamol esperada, considerando o valor declarado $(750 \mathrm{mg} / \mathrm{com}-$ primido) e o peso médio dos comprimidos (\% paracetamol esperada $=0,75 \mathrm{~g} /$ massa média dos comprimidos $(\mathrm{g}) \mathrm{x} 100)$.

\section{Obtenção dos espectros NIR e pré-processamento}

Os espectros NIR foram obtidos em um espectrofotômetro NIR $\left(\right.$ Perkin Elmer ${ }^{\circledR}$ ) com acessório para obtenção de espectros de refletância difusa. Cada frasco contendo as amostras foi posicionado no acessório de refletância difusa (Figura 1S) e os espectros de \% de reflectância (\% R) na faixa espectral de 10.000 a $4.000 \mathrm{~cm}^{-1}$ foram obtidos com média de 16 varreduras. No Matlab, os espectros NIR obtidos para os conjuntos de calibração, validação e medicamentos foram transformados de \% R para log (1/R), o qual é proporcional à concentração do analito e por este motivo deve ser utilizado nas etapas de calibração. Os espectros foram pré-processados empregando o método MSC.

\section{Softwares e algoritmos}

Para os cálculos descritos neste tutorial foi utilizado um pacote gratuito para aplicação do algoritmo PLS disponibilizado pelo grupo de Quimiometria da Universidade Livre de Bruxelas (Chemometrics in Analytical Chemistry, Chemo AC) no website: http://www.vub. ac.be/fabi/publiek/index.html. Os algoritmos foram executados em ambiente Matlab 7.0. O pacote disponibilizado contém algoritmos para a realização do pré-processamento MSC e centra as matrizes X e Y na média de forma automática.

\section{RESULTADOS E DISCUSSÃO}

Inicialmente foi avaliado o espectro do paracetamol na região NIR, em comparação com o espectro do placebo. O paracetamol, como a maioria dos fármacos, apresenta ligações C-H, N-H e O-H, o que faz com que seu espectro seja intenso na região NIR, conforme é possível observar na Figura 1.

Também é possível observar na Figura 1 que o placebo apresenta bandas relativas a vibrações das ligações em muitas regiões comuns ao paracetamol, ou seja, existe superposição espectral. Este fato impede a calibração univariada e requer um método de calibração multivariada.

A seguir serão mostrados os passos a serem seguidos para o desenvolvimento do modelo PLS, validação e previsão do teor de paracetamol nos medicamentos comerciais empregando o programa Matlab. Como sugestão, antes da realização do PLS, o aluno poderá realizar uma análise exploratória empregando PCA, uma vez que o mesmo foi descrito no Tutorial I. ${ }^{1}$ Além disso, outra sugestão seria explorar o mesmo conjunto de dados empregando a Regressão em Componentes Principais (PCR), cujos algoritmos também se encontram disponíveis para download a partir do site indicado. Os resultados deverão ser equivalentes. Os espectros utilizados neste tutorial estão

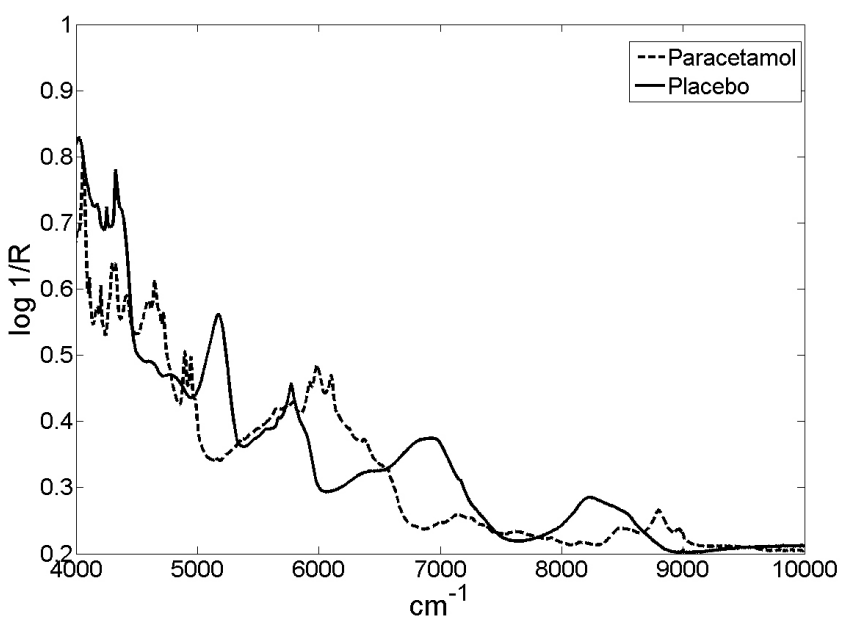

Figura 1. Espectros do paracetamol e do placebo no formato log $1 / R$

prontamente disponíveis aos interessados por meio de contato com o autor R. J. Poppi (ronei@iqm.unicamp.br).

Carregar os espectros e criar as matrizes:

(1) Carregar os espectros do conjunto de calibração, de validação e dos medicamentos comerciais no Matlab digitando o comando load. Por exemplo,

>>load C11.ASC. A denominação C11 indica calibração amostra 1 , replicata $1 ; \mathrm{C} 12$ indica calibração amostra 1 , replicata 2 e assim por diante. As amostras de validação são indicadas pela inicial "V" e os medicamentos pelas iniciais "FAB".

(2) Criar as matrizes de espectros do conjunto de calibração, validação e medicamentos.

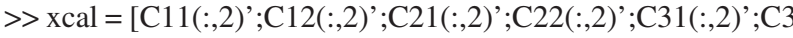
$2(:, 2)^{\prime} ; \mathrm{C} 41(:, 2)^{\prime} ; \mathrm{C} 42(:, 2)^{\prime} ; \mathrm{C} 51(:, 2)^{\prime} ; \mathrm{C} 52(:, 2)^{\prime} ; \mathrm{C} 61(:, 2)^{\prime} ; \mathrm{C} 62(:$, 2)';C71(:,2)';C72(:,2)';C81(:,2)';C82(:,2)'];

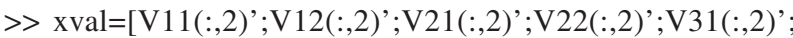
V32(:,2)'; V41(:,2)';V42(:,2)';V51(:,2)';V52(:,2)'];

$>>$ xmed $=\left[\right.$ FAB11 $(:, 2)^{\prime} ;$ FAB12(:,2)';FAB21(:,2)';FAB22(:,2)'; FAB31(:,2)'; FAB32(:,2)';FAB41(:,2)';FAB42(:,2)'];

(3) Criar os vetores $\mathbf{y}$ contendo as concentrações de paracetamol para as amostras de calibração, validação e medicamentos: >>ycal=[59.82;59.82;64.84;64.84;70.00;70.00;75.04;75.04;80. $04 ; 80.04 ; 84.90 ; 84.90 ; 89.40 ; 89.40 ; 94.49 ; 94.49]$; >>yval=[63.07; 63.07; 68.56;68.56; 73.10; 73.10; 88.04; 88.04; $83.41 ; 83.41]$; >>ymed=[89.83;89.83;88.67;88.67;90.91;90.91;90.42;90.42];

(4) Criar um vetor de número de onda para ser utilizado como eixo $\mathrm{x}$ nos gráficos:

$>$ num $=\mathrm{C} 11(:, 1)^{\prime}$;

Criar e formatar os gráficos:

Serão descritos abaixo os comandos para gerar os espectros e formatá-los no próprio Matlab, sem o auxílio de nenhum toolbox adicional. Serão mostrados comandos para gerar figuras contendo todos os espectros juntos (calibração, validação e medicamentos), bem como cada um de forma separada. A análise de todos os espectros de forma conjunta permite avaliar se existe alguma discrepância entre as amostras.

(5) Gerar um gráfico contendo todos os espectros das amostras de calibração, de validação e dos medicamentos em porcentagem 
de refletância (\% R), conforme mostrado na Figura 2S:

$>$ espectros $=[$ xcal; $x$ val; $x$ med $]$;

$>$ figure (1)

$>$ set $(0$, 'defaultAxesFontName', 'arial')

$>$ axes('FontSize',24);

$>$ plot(num,espectros)

>> xlabel('cm^^^1','FontSize',30,'FontName','arial');

>> ylabel('Reflectância (\%)','FontSize',30,'FontName','arial');

(6) Gerar e formatar os gráficos individuais para as amostras dos conjuntos de calibração, de validação e dos medicamentos (Figuras $3 \mathrm{~S}, 4 \mathrm{~S}$ e $5 \mathrm{~S})$ :

$>$ figure (2)

$>$ set( 0 ,'defaultAxesFontName', 'arial')

$>$ axes('FontSize',24);

$>$ plot(num,xcal)

>>xlabel('cm^^1','FontSize',30,'FontName','arial');

>>ylabel('Reflectância (\%) ','FontSize',30,'FontName','arial');

$>$ figure (3)

$>$ axes('FontSize',24);

$>$ plot(num,xval)

> >xlabel('cm^^^1','FontSize',30,'FontName','arial');

>>ylabel('Reflectância (\%)','FontSize',30,'FontName','arial');

$>$ figure (4)

$>$ axes('FontSize',24);

$>$ plot(num,xmed)

> >xlabel('cm^^^1','FontSize',30,'FontName','arial');

> >ylabel('Reflectância (\%)','FontSize',30,'FontName','arial');

(7) Transformar o sinal de porcentagem de reflectância (\% R) para $\log 1 / \mathrm{R}$, o qual é proporcional à concentração.

$>$ >xcalt $=\log 10(1 . /($ xcal. $/ 100))$;

$>>$ xvalt $=\log 10(1 . /($ xval. $/ 100))$

$>>$ xmedt $=\log 10(1 . /($ xmed./100) $)$;

O "ponto" nas operações acima indica que a divisão deve ser realizada de forma individual para cada variável (comprimento de onda) do espectro.

(8) Criar um único gráfico com os espectros dos conjuntos de calibração, de validação e dos medicamentos transformados para log 1/R, conforme mostrado na Figura 2:

$>$ espectrost $=[$ xcalt; $x$ valt; $x$ medt $]$;

$>$ figure (5)

$>$ axes('FontSize',24);

$>$ plot(num,espectrost)

> xlabel('cm^^^1','FontSize',30,'FontName','arial');

>>ylabel('log 1/R','FontSize',30,'FontName','arial');

(9) Criar e formatar os gráficos individuais com os espectros das matrizes de calibração, de validação e dos medicamentos transformadas para $\log (1 / \mathrm{R})$ (Figura $6 \mathrm{~S}, 7 \mathrm{~S}$ e $8 \mathrm{~S}$, respectivamente): $>$ figure (6)

$>$ set(0,'defaultAxesFontName', 'arial')

$>$ axes('FontSize',24);

$>$ plot(num,xcalt)

> >xlabel('cm^^^1','FontSize',30,'FontName','arial');

> >ylabel('log 1/R','FontSize',30,'FontName','arial');

$>$ figure (7)

$>$ axes('FontSize',24);

$>$ plot(num,xvalt)

>>xlabel('cm^^1','FontSize',30,'FontName','arial');

>>ylabel('log 1/R','FontSize',30,'FontName','arial');

$>$ >figure (8)

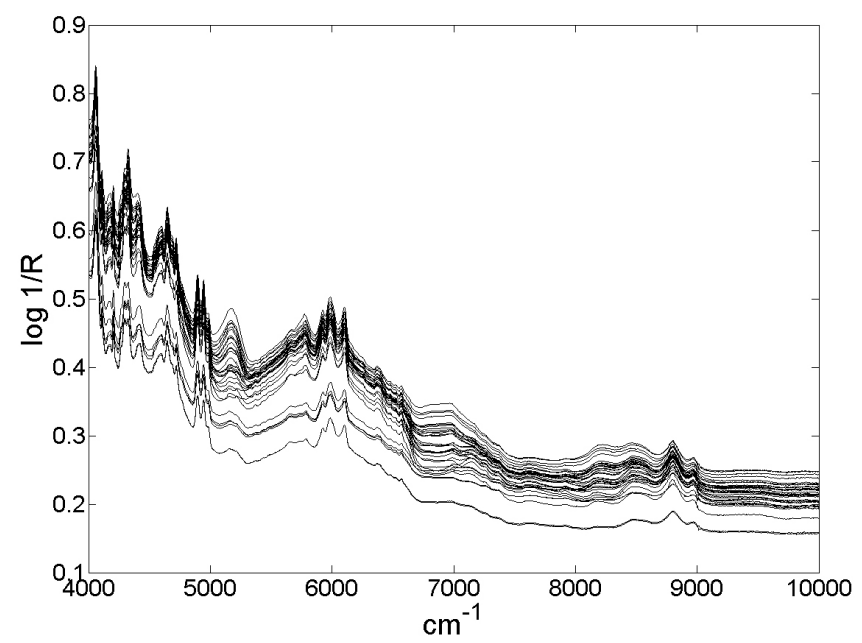

Figura 2. Conjunto dos espectros das amostras de calibração, validação e dos medicamentos em log $(1 / R)$

>>axes('FontSize',24);

$>$ plot(num,xmedt)

>>xlabel('cm^^^1','FontSize',30,'FontName','arial');

>>ylabel('log 1/R','FontSize',30,'FontName','arial');

Selecionar a faixa espectral de interesse:

(10) Selecionar a faixa espectral de 4001 a $5000 \mathrm{~cm}^{-1}$, o que corresponde às variáveis 5000:6001. Esta região tem forte correlação com o fármaco e por este motivo foi utilizada para tornar o modelo mais robusto à previsão da concentração do mesmo nos medicamentos, uma vez que os medicamentos comerciais apresentam variações em relação aos excipientes. Foi verificado que esta região é robusta para previsão do fármaco mesmo na presença dessas diferenças.

$>$ xcalt_c $=$ xcalt $(:, 5000: 6001)$;

$\gg$ xvalt_c $=$ xvalt $(:, 5000: 6001)$

$>$ >xmedt_c $=$ xmedt $(:, 5000: 6001)$;

$>$ num_c=num(:,5000:6001);

(11)Criar um gráfico com os espectros dos conjuntos de calibração, de validação e dos medicamentos na faixa espectral de interesse (Figura 9S).

$>>$ espectrostc $=[$ xcalt_c; xvalt_c;xmedt_c];

$>$ figure (9)

$>$ set( 0 ,'defaultAxesFontName', 'arial')

$>$ axes('FontSize',24);

$>$ plot(num_c,espectrostc)

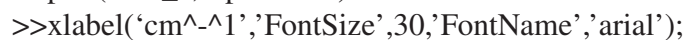

> ylabel('log 1/R','FontSize',30,'FontName','arial');

(12) Criar gráficos com os espectros individuais dos conjuntos de calibração, de validação e dos medicamentos na faixa espectral de interesse (Figuras 10S, 11S e 12S, respectivamente):

$>$ figure (10)

$>>\operatorname{set}(0$, 'defaultAxesFontName', 'arial')

$>$ axes('FontSize',24);

$>$ plot(num_c,xcalt_c)

>>xlabel('cm^^^1','FontSize',30,'FontName', 'arial');

>>ylabel(' $\log 1 / \mathrm{R}$ ','FontSize',30,'FontName','arial');

$>>$ figure (11)

$>$ axes('FontSize',24);

$>$ plot(num_c,xvalt_c)

>>xlabel('cm^^^1','FontSize',30,'FontName','arial'); 
>>ylabel('log 1/R','FontSize',30,'FontName','arial');

$>>$ figure (12)

> axes('FontSize',24);

$>$ plot(num_c,xmedt_c)

>>xlabel( 'cm^^^1','FontSize',30,'FontName','arial');

> >ylabel('log 1/R','FontSize',30,'FontName','arial');

Pré-processamento:

(13) Aplicar o algoritmo para correção de espalhamento (MSC) nas matrizes individuais dos conjuntos de calibração, de validação e dos medicamentos. Este procedimento é necessário para remover flutuações de linha base que estão relacionadas com aspectos físicos das amostras, tais como tamanho e forma das partículas e não com a composição química das mesmas.

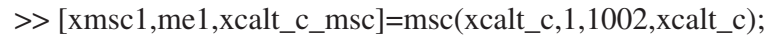

$>$ >xmsc2,me2,xvalt_c_msc $]=m s c\left(x c a l t \_c, 1,1002\right.$, ,xvalt_c);

$>$ >xmsc3,me3,xmedt_c_msc] $=\operatorname{msc}\left(\right.$ xcalt_c, $\left.1,1002, x m e d t \_c\right)$;

(14) Aplicar o algoritmo para correção de espalhamento (MSC) na matriz contendo os conjuntos de espectros de calibração, de validação e dos medicamentos.

$>$ > $[$ mmsc4,me4,espectrostc_msc $]=$ msc(espectrostc, 1,1002 ,espe ctrostc);

(15) Criar o gráfico dos espectros das amostras de calibração, validação e dos medicamentos após a aplicação do MSC, conforme mostrado na Figura 3:

$>>$ figure (13)

$>$ set( 0 ,'defaultAxesFontName', 'arial')

$>$ axes('FontSize', 24);

$>>$ plot(num_c,espectrostc_msc,'k')

>>xlabel('cm^^^1','FontSize',30,'FontName','arial');

>>ylabel('log 1/R','FontSize',30,'FontName','arial');

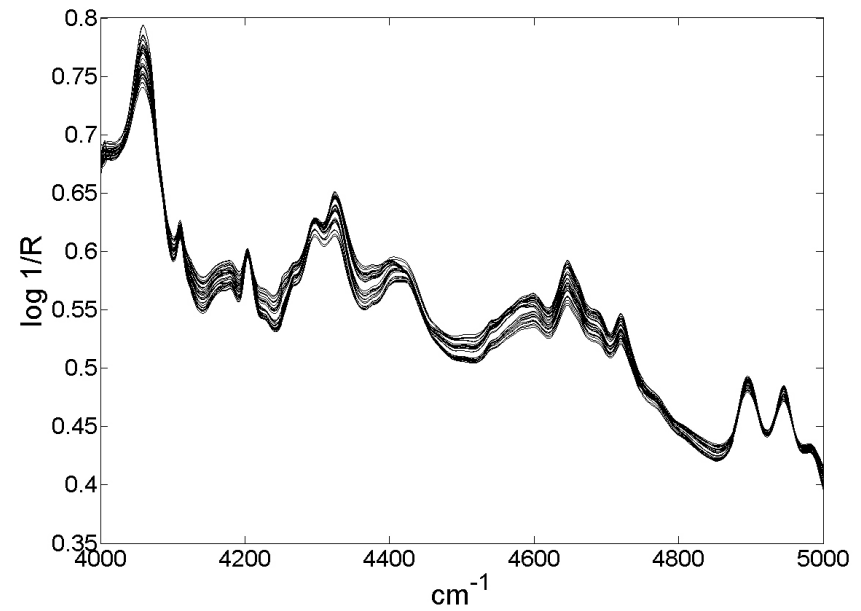

Figura 3. Conjunto dos espectros das amostras de calibração, validação e dos medicamentos após seleção da região espectral de interesse e correção por MSC

(16) Criar os gráficos com os espectros individuais das amostras de calibração, de validação e dos medicamentos após aplicação do MSC (Figuras 13S, 14S e 15S, respectivamente).

$>>$ figure (14)

$>$ set( 0 ,'defaultAxesFontName', 'arial')

$>$ axes('FontSize',24);

$>>$ plot(num_c,xcalt_c_msc)

>>xlabel( 'cm^^^1','FontSize',30,'FontName','arial'); >>ylabel('log 1/R','FontSize',30,'FontName','arial');

$>$ figure (15)

$>$ axes ('FontSize',24);

$>$ plot(num_c,xvalt_c_msc)

> >xlabel( 'cm^^1','FontSize',30,'FontName','arial');

>>ylabel('log 1/R','FontSize',30,'FontName','arial');

$>$ figure (16)

$>$ axes('FontSize',24);

$>>$ plot(num_c,xmedt_c_msc)

> >xlabel( 'cm^^^11','FontSize',30,'FontName','arial');

>>ylabel('log 1/R','FontSize',30,'FontName','arial');

Validação cruzada:

(17) Para determinar o número de variáveis latentes a serem empregadas no modelo, deve ser realizado o procedimento de validação cruzada, conforme mostrado a seguir:

$>>$ [rmsecv,yh,e] $=$ plscv(xcalt_c_msc,ycal, 0,10$)$;

(18) Criar o gráfico de RMSECV vs número de variáveis latentes, conforme mostrado na Figura 4. Neste ponto o usuário deverá digitar o número de variáveis latentes com base no mínimo da curva gerada. No exemplo mostrado na Figura 4, o número de variáveis latentes é 3, uma vez que a partir deste número não existe variação apreciável no valor de RMSECV.

$>$ figure (17)

$>$ set(0,'defaultAxesFontName', 'arial')

$>$ axes ('FontSize',24);

$>$ plot(rmsecv,'k'); hold on

$>$ plot(rmsecv,'ko')

> >ylabel('RMSECV','FontSize',30,'FontName','arial');

>>xlabel('Variáveis latentes','FontSize',30,'FontName','arial'); >>vl=input('Quantas variaveis latentes?'); Entrar com o numero de variáveis latentes e teclar o <enter>.

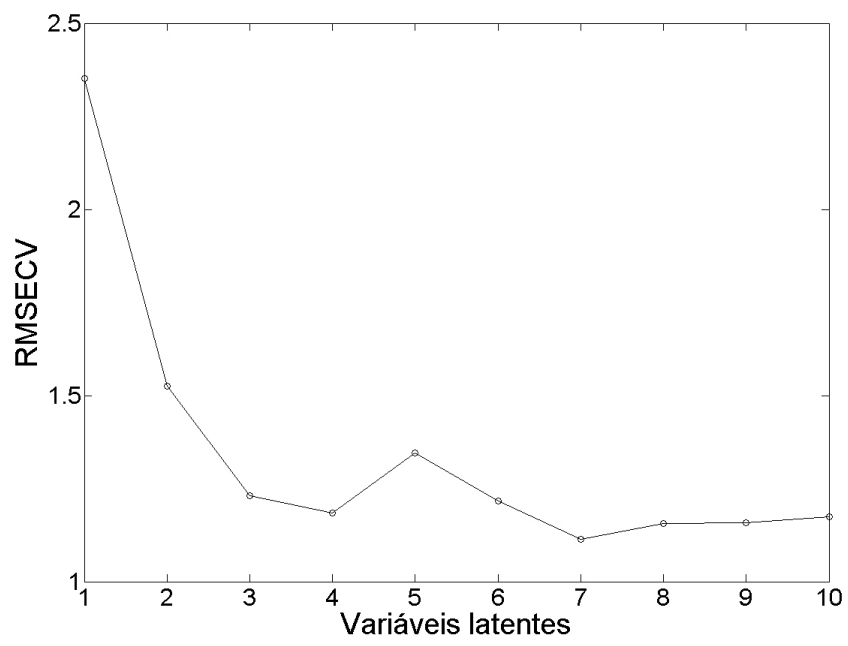

Figura 4. Gráfico do número de variáveis latentes vs o valor de RMSECV

Calibração:

(19) Nesta etapa o algoritmo irá fazer a previsão do teor de paracetamol para as amostras de calibração sem retirá-las do modelo (vetor ypred1) e calculará os valores de RMSEC com o número de variáveis latentes indicadas anteriormente.

$>$ > [ypred1] $=$ plspred(xcalt_c_msc,ycal,xcalt_c_msc,0,vl);

$>>\operatorname{rmsec}=\operatorname{sqrt}((\operatorname{sum}(($ ycal-ypred1).^2) $) /($ length $($ ycal $)-\mathrm{vl}-1))$;

(20)Construir o gráfico de valores esperados versus valores previstos pelo modelo PLS para as amostras de calibração (Figura 16S) 
$>>$ figure (18)

$>$ set(0,'defaultAxesFontName', 'arial')

$>$ axes('FontSize',24);

$>>$ r=corrcoef $($ ycal,ypred 1$)$

$>$ >bias $=$ sum(ycal-ypred1)/length(ycal);

$>\mathrm{s} 1=\operatorname{sprintf}\left(\right.$ ' $\left.\mathrm{r} \wedge 2 \mathrm{c}=\% 0.4 \mathrm{f}^{\prime}, \mathrm{r}(1,2)^{\wedge} 2\right)$;

$>\mathrm{s} 2=\operatorname{sprintf}($ 'rmsec $=\% 0.4 \mathrm{f}$ ', $\mathrm{rmsec}$ );

$>>$ s $3=\operatorname{sprintf}$ ('bias $=\% 0.4 \mathrm{f}$ ', bias);

>>plot(ycal,ypred1,'ko','LineWidth',2,'MarkerSize',15,'Marker

FaceColor',[.8 .8 .8]); Isline

$>>$ pa1 $=\min (\mathrm{ycal}) ; \mathrm{pa} 2=\max (\mathrm{ycal})$;

$>>$ axis([.95*pa1 $1.05 *$ pa2 $.95 *$ pa1 $1.05 *$ pa2] $)$;

> text(pa1-abs(pa1*0.04),1.02*pa2-abs(pa2*0.05),s1,'FontSize',

20,'FontName','Arial');

>>text(pa1-abs(pa1*0.04),1.02*pa2-abs(pa2*0.08),s2,'FontSize',

20,'FontName','Arial');

> text(pa1-abs(pa1*0.04),1.02*pa2-abs(pa2*0.11),s3,'FontSize',

20,'FontName','Arial');

>>ylabel('Valores previstos (\%)','FontSize',30,'FontName', 'arial');

>>xlabel('Valores esperados (\%)','FontSize',30,'FontName', 'arial');

Validação Externa

(21) Nesta etapa, o modelo de calibração será avaliado (validado) utilizando um conjunto externo de amostras. O vetor ypred2 contém os valores previstos de paracetamol para as amostras de validação.

$>>$ ypred2]=plspred(xcalt_c_msc,ycal,xvalt_c_msc,0,3);

(22)Calcular o valor de RMSEV de validação: $>\operatorname{rmsev}=\operatorname{sqrt}((\operatorname{sum}(($ yval-ypred2).^2) $) /($ length $($ yval $)))$

(23)Construir o gráfico de valores previstos versus os valores esperados para as amostras de validação externa (Figura 17S):

$>$ figure (19)

$>$ set( 0 ,'defaultAxesFontName', 'arial')

$>$ axes('FontSize',24);

$>>$ r=corrcoef(yval,ypred2);

$>$ biasv=sum (yval-ypred2)/length (yval);

$>\mathrm{s} 12=\operatorname{sprintf}\left(\right.$ ' $\left.\mathrm{r}^{\wedge} 2 \mathrm{v}=\% 0.4 \mathrm{f}^{\prime}, \mathrm{r}(1,2)^{\wedge} 2\right)$;

$>>$ s22 $=\operatorname{sprintf('rmsev~}=\% 0.4 \mathrm{f}$ ',rmsev);

$>>$ s32 $=\operatorname{sprintf}($ 'biasv $=\% 0.4 \mathrm{f}$ ', biasv);

>>plot(yval,ypred2,'g*','LineWidth',2,'MarkerSize',15,'Marker

FaceColor',[.8 .8 .8]);1sline

$>>\mathrm{pa} 3=\min (\mathrm{yval}) ; \mathrm{pa} 4=\max (\mathrm{yval})$

>>text(pa3-abs(pa3*0.04),1.02*pa4- abs(pa4*0.05),s12,'FontSize',

20,'FontName','Arial');

$>$ text(pa3-abs(pa3*0.04),1.02*pa4-abs(pa4*0.08),s22,'FontSize',

20,'FontName','Arial');

>>text(pa3-abs(pa3*0.04),1.02*pa4-abs(pa4*0.11),s32,'FontSize',

20,'FontName','Arial');

>>ylabel('Valores previstos (\%)','FontSize',30,'FontName', 'arial');

>>xlabel('Valores esperados (\%)','FontSize',30,'FontName', 'arial');

(24)Construir os gráficos de valores esperados versus valores previstos para as amostras de calibração e de validação externa em conjunto (Figura 5):

$>>$ figure (20)

$>>\operatorname{set}(0$, 'defaultAxesFontName', 'arial')

$>$ axes('FontSize', 24); >>plot(ycal,ypred1,'ko',yval,ypred2,'k*', 'LineWidth', 2,'Marker

Size',15,'MarkerFaceColor',[.8 .8 .8]);

>>legend( 'Calibração','Validação Externa');

$>$ axis([.95*pa1 $1.05 *$ pa2 $.95 *$ pa1 $1.05 *$ pa2] $)$;

$>$ h=refline $(1,0)$;set(h,'color','k');

>>text(pa1-abs(pa1*0.04),1.1*pa4-abs(pa4*0.05),s12,'FontSize',

20,'FontName','Arial');

>>text(pa1-abs(pa1*0.04),1.1*pa4-abs(pa4*0.08),s22,'FontSize',

20,'FontName','Arial');

>>text(pa1-abs(pa1*0.04),1.1*pa4-abs(pa4*0.11),s32,'FontSize',

20,'FontName','Arial');

>>text(pa1-abs(pa1*0.04),1.04*pa2-abs(pa2*0.16),s1,'FontSize',

20,'FontName','Arial');

>>text(pa1-abs(pa1*0.04),1.04*pa2-abs(pa2*0.19),s2,'FontSize', 20,'FontName','Arial');

>>text(pa1-abs(pa1*0.04),1.04*pa2-abs(pa2*0.22),s3,'FontSize', 20,'FontName','Arial');

>>ylabel('Valores previstos (\%)','FontSize',30,'FontName', 'arial');

>>xlabel('Valores esperados (\%)','FontSize',30,'FontName', 'arial');

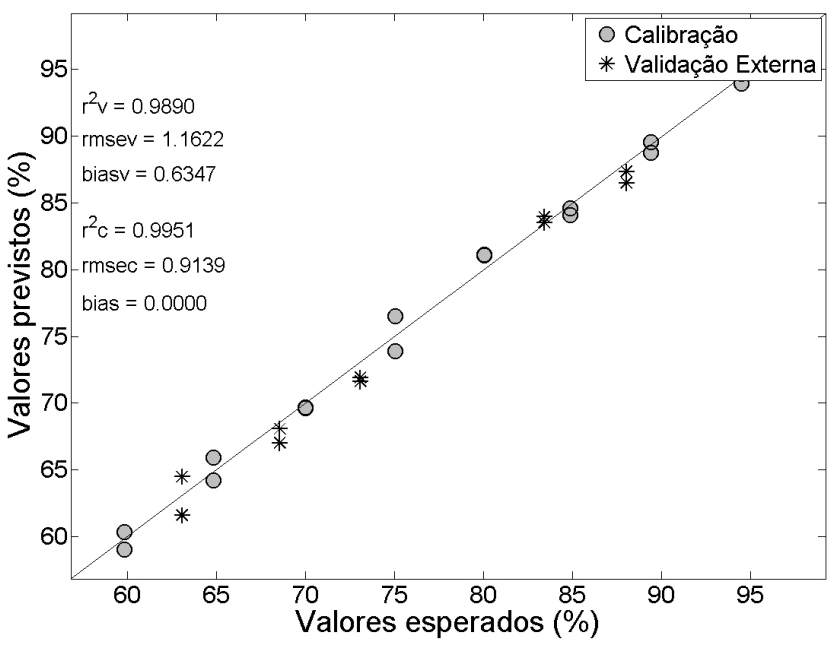

Figura 5. Gráfico dos valores esperados vs valores previstos para a calibração e validação externa

Como é possível observar na Figura 5, os valores de RMSEC (amostras de calibração) e RMSEV (validação externa) são, respectivamente, 0,9 e $1,2 \%$. A unidade destas grandezas é a mesma da propriedade de interesse $(\% \mathrm{~m} / \mathrm{m}$ paracetamol) e assim permite uma avaliação do erro médio associado na previsão por PLS. As amostras encontram-se todas próxima à reta e os valores dos coeficientes de determinação para a calibração e validação foram 0,995 e 0,989, respectivamente, indicando excelente concordância entre os valores esperados e aqueles previstos pelo modelo PLS.

Previsão da porcentagem de paracetamol nos medicamentos comerciais:

(25) Os valores previstos de paracetamol nos medicamentos comerciais serão mostrados no vetor ypred3:

> $[$ ypred3]=plspred(xcalt_c_msc,ycal,xmedt_c_msc,0,3);

(26)Calcular do RMSEP para as amostras de medicamentos: $>>\operatorname{rmsep}=\operatorname{sqrt}((\operatorname{sum}(($ ymed-ypred3).^2) $) /($ length $($ ymed $)))$

(27)Criar um gráfico com os valores esperados $v s$ valores previstos pelo PLS, conforme mostrado na Figura 6: 
$>>$ figure (21)

$>$ set(0,'defaultAxesFontName', 'arial')

$>$ axes('FontSize',24);

$>$ h1=bar([ypred3 ymed]);colormap gray

>>legend('Valores previstos (\%)','Valores esperados (\%)')

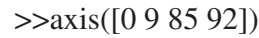

> >xlabel('Amostras','FontName','arial','FontSize',30);

>>ylabel('Teores de paracetamol (\%)','FontSize',30,'FontName',

'arial');

>>hline (85,'k')

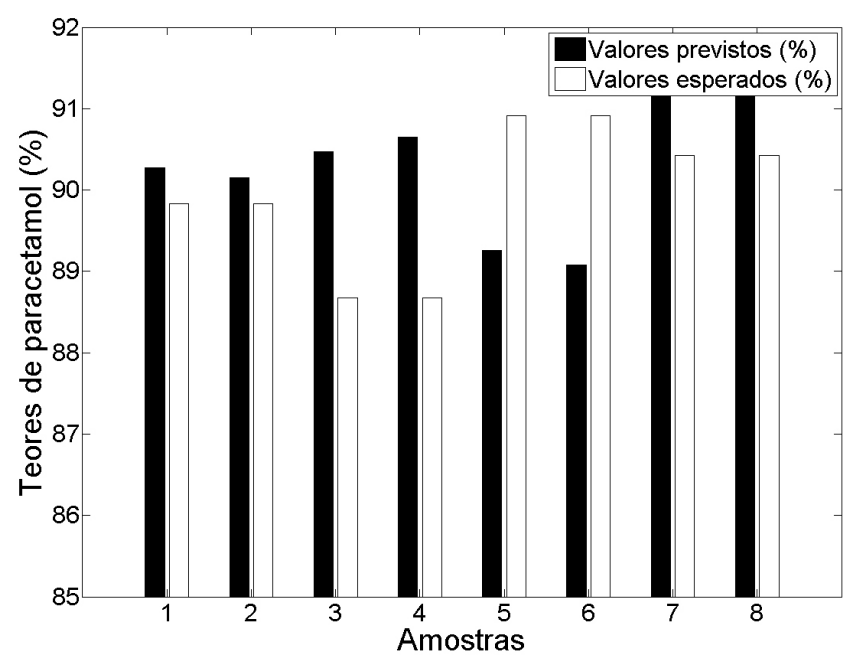

Figura 6. Valores esperados vs valores previstos para os comprimidos comerciais pelo $P L S$

O valor de RMSEP encontrado para os medicamentos comercial neste exemplo foi de 1,4\%. Vale ressaltar que a concentração do paracetamol em comprimidos comerciais é próxima de $90 \%$ (m/m), o que permite que a previsão seja realizada mesmo se houver pequenas diferenças nos excipientes utilizados nos medicamentos comerciais em relação aos utilizados na calibração. Além disso, a seleção da região espectral descrita torna a previsão mais robusta em relação à variação dos excipientes.

Quando o medicamento (produto final) contém monografia na Farmacopéia, os limites de aceitação do teor do fármaco no medicamento são estabelecidos nesta. A monografia "Paracetamol comprimidos" encontrada na Farmacopéia Brasileira ${ }^{20}$ estabelece que o fármaco deva estar na faixa de 95 a $105 \%$ do valor declarado no rótulo. Como este valor é de $750 \mathrm{mg} / \mathrm{comprimido}$, a faixa de valores aceitos seria de 712,5 a 787,5 mg/comprimido. Considerando o erro de previsão de $1,4 \%$ e o valor médio previsto nos medicamentos $(90,04 \%)$, a faixa de valores previstos seria de 739,5 a 760,5 a qual está dentro da faixa permitida pela Farmacopéia brasileira.

\section{CONCLUSÃO}

Neste trabalho foi apresentado um procedimento e foram indicados todos os comandos necessários para a construção, validação e aplicação de um modelo de calibração multivariada por PLS como sugestão de experimento didático para a graduação, pós-graduação ou iniciantes em quimiometria. Os algoritmos utilizados podem ser obtidos gratuitamente no site http://www.vub.ac.be/fabi/publiek/ index.html. Os comandos de formatação das figuras também foram descritos de tal maneira que o usuário não necessite de nenhum toolbox adicional para realizar os cálculos empregando o programa Matlab. O experimento é simples em relação ao preparo das amostras e obtenção dos espectros e tem demonstrado ser adequado para a sua aplicação em disciplinas experimentais de laboratório com 4 horas de duração.

Esse experimento demonstra aos alunos a possibilidade de quantificação do analito na amostra sem necessidade de separação prévia, mesmo na presença de espécies interferentes (excipientes), o que só é possível mediante o uso de calibração multivariada. Com a seleção da região espectral de interesse e pelo fato do teor do fármaco ser alto nos medicamentos (próximo de $90 \% \mathrm{~m} / \mathrm{m}$ ), a previsão do PLS é bastante concordante em relação aos valores nominais indicados pelos fabricantes. A etapa de tratamento dos dados no Matlab pode ser realizada no final da aula experimental, em uma sala de computadores. Nesta etapa, os alunos podem ser divididos em grupos sem o comprometimento da qualidade da aula, e o tempo estimado de duração do tratamento de dados é de aproximadamente 1 hora. Além disso, o tutorial também oferece a oportunidade de executar o PLS passo a passo para iniciantes em quimiometria com ou sem a supervisão de um professor ou especialista em Matlab.

\section{MATERIAL SUPLEMENTAR}

No material suplementar, disponível em http://quimicanova. sbq.org.br, na forma de arquivo PDF, com acesso livre, a Figura 1S apresenta o acessório de reflectância difusa do espectrofotômetro NIR. A Figura $2 \mathrm{~S}$ apresenta o conjunto dos espectros das amostras de calibração, validação e medicamentos em \% de reflectância. Nas Figuras 3S, 4S e 5S, os conjuntos de espectros das amostras de calibração, de validação e dos medicamentos em \% de reflectância são apresentados, respectivamente. Nas Figuras $6 \mathrm{~S}, 7 \mathrm{~S}$ e $8 \mathrm{~S}$, os conjuntos de espectros das amostras de calibração, de validação e dos medicamentos em $\log (1 / \mathrm{R})$ são apresentados, respectivamente. A Figura 9S apresenta o conjunto dos espectros em $\log 1 / \mathrm{R}$ após seleção da região espectral de interesse. As Figuras 10S, 11S e 12S apresentam, respectivamente, o conjunto de espectros das amostras de calibração, de validação e dos medicamentos em $\% \log 1 / \mathrm{R}$ na faixa espectral selecionada. Nas Figuras 13S, 14S e 15S, os conjuntos de espectros das amostras de calibração, de validação e dos medicamentos após correção por MSC, são apresentados, respectivamente. A Figura 16S apresenta o gráfico dos valores esperados versus os valores previstos do modelo de calibração e finalmente, a Figura $17 \mathrm{~S}$ apresenta o gráfico dos valores esperados versus os $v s$ valores previstos pelo modelo para validação externa.

\section{TRABALHOS FUTUROS}

Dando continuidade à série de tutoriais em Quimiometria, o próximo tutorial estará relacionado a um experimento de planejamento e otimização das condições experimentais para determinação de $\mathrm{Fe}^{2+}$ em medicamentos, empregando o-fenantrolina como complexante e espectroscopia na região do visível. O objetivo é mostrar aos alunos que os métodos multivariados de planejamento geram informações valiosas sobre o sistema em estudo, com um número reduzido de experimentos. A partir do ajuste de um modelo aos dados, será construída a superfície de resposta, a qual mostra como varia a propriedade de interesse conforme as condições experimentais são alteradas. No exemplo elaborado, existe interação entre as variáveis e, dessa maneira, a otimização univariada não seria efetiva ou seria demasiadamente trabalhosa.

\section{AGRADECIMENTOS}

Os autores agradecem ao Programa Estágio Docente (PED) da Unicamp, aos doutorandos G. P. Sabin e M. B. M. Lopez pelas contribuições, e ao constante apoio do técnico do LAQQA, H. Machado. 
Agradecemos também aos órgãos de fomento à pesquisa: CAPES, CNPq e FAPESP.

\section{REFERÊNCIAS}

1. Souza, A. M.; Poppi, R. J.; Quim. Nova 2012, 35, 223.

2. Ciurczak, E. W. Em Handbook of Near-Infrared Analysis; Burns, D. A.; Ciurczak E. W., eds.; Marcel Dekker, INC.: New York, 2001, chap 2.

3. Williams, P.; Norris, K. H.; Near infrared technology in the agricultural and food industries, Amer. Assoc. of Cereal Chemists: St. Paul, 1987.

4. Workman, J. J.; Weyer, L.; Practical Guide to interpretative NearInfrared spectroscopy, Taylor \& Francis: Boca Raton, 2008.

5. McClure, W. F.; Anal. Chem. 1994, 66, 43A.

6. Bosco, G.; TrAC, Trends Anal. Chem. 2010, 29, 197.

7. Pasquini, C.; J. Braz. Chem. Soc. 2003, 14, 198.

8. Brereton, R. G.; Analyst 2000, 125, 2125.

9. Rinnan, A.; Van den Berg, F.; Engelsen, S. B.; TrAC, Trends Anal. Chem. 2009, 28, 1201.

10. Martens, H.; Naes, T.; Multivariate Calibration, $2^{\text {nd }}$ ed., John Wiley \& Sons: Chichester, 1993.
11. Beebe, K. R.; Pell, R. J.; Seasholtz, M. B.; Chemometrics: A Practical Guide; John Wiley \& Sons: New York, 1998.

12. Geladi, P.; Kowalski, B. R.; Anal. Chim. Acta 1986, 185, 1.

13. Lorber, A.; Wangen, L. E.; Kowalski, B. R.; J. Chemom. 1987, 1, 19.

14. Andersson, M.; J. Chemom. 2009, 23, 518.

15. ASTM - American Society for Testing and Materials; Standard Practices for Infrared Multivariate Quantitative Analysis, ASTM E 1655, 2005.

16. Roggo, I.; Chalus, P.; Maurer, L.; Lema-Martinez, C.; Edmond, A.; Jent, N.; J. Pharm. Biomed. Anal. 2007, 44, 683.

17. De Bleye, C.; Chavez, P. F.; Mantanus, J.; Hubert, P.; Rozet, E.; Ziemons, E.; J. Pharm. Biomed. Anal. 2012, 69, 125.

18. Yappert, M. C.; J. Chem. Educ. 1999, 76, 315.

19. Ferreira, M. M. C.; Antunes, A. M.; Melgo, M. S.; Volpe, P. L. O.; Quim. Nova $1999,22,724$

20. http://www.anvisa.gov.br/hotsite/cd_farmacopeia/pdf/volume2.pdf, acessado em Dezembro 2012. 


\section{EXPERIMENTO DIDÁTICO DE QUIMIOMETRIA PARA CALIBRAÇÃO MULTIVARIADA NA DETERMINAÇÃO DE PARACETAMOL EM COMPRIMIDOS COMERCIAIS UTILIZANDO ESPECTROSCOPIA NO INFRAVERMELHO PRÓXIMO: UM TUTORIAL, PARTE II}

André Marcelo de Souza\#, Márcia Cristina Breitkreitz, Paulo Roberto Filgueiras, Jarbas José Rodrigues Rohwedder e Ronei Jesus Poppi*

Departamento de Química Analítica, Instituto de Química, Universidade Estadual de Campinas, CP 6154, 13084-971 Campinas - SP, Brasil
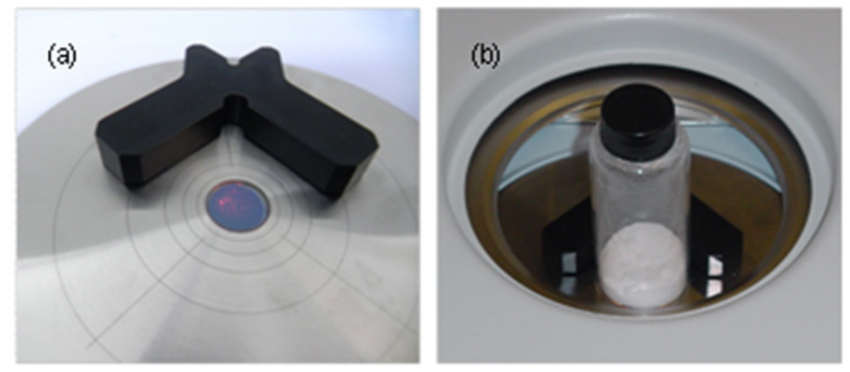

Figura 1S. Acessório de reflectância difusa do espectrofotômetro NIR utilizado no experimento: (a) Foto da janela de saída do feixe de radiação; (b) posicionamento da amostra no feixe de radiação para a aquisição dos espectros

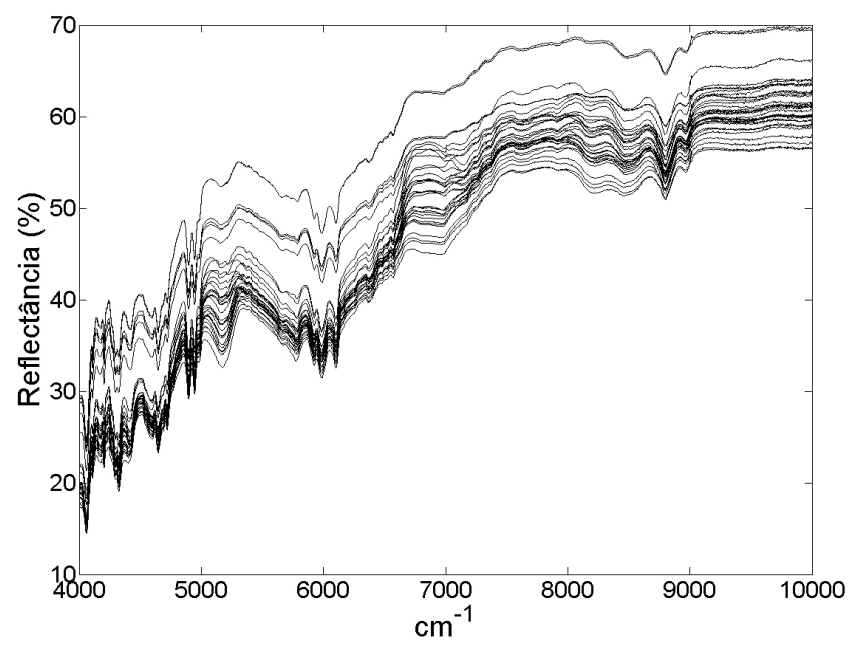

Figura 2S. Conjunto dos espectros das amostras de calibração, validação e dos medicamentos em \% de reflectância

*e-mail: ronei@iqm.unicamp.br

\#Endereço atual:Embrapa Solos, Empresa Brasileira de Pesquisa Agropecuária - Rua Jardim Botânico, 1.024 - Jardim Botânico - CEP 22460-000 - Rio de Janeiro- RJ - Brasil.

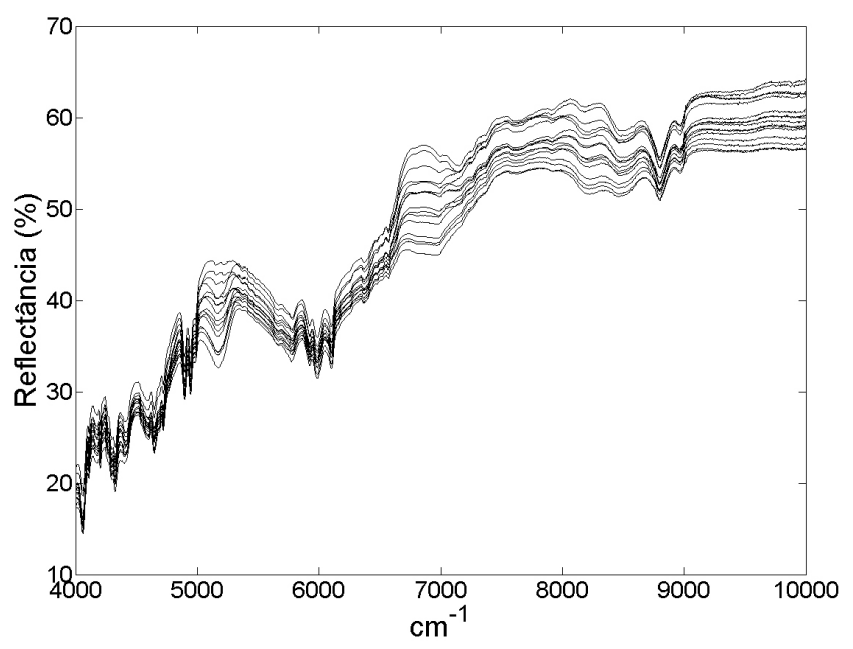

Figura 3S. Conjunto de espectros das amostras de calibração em \% de reflectância

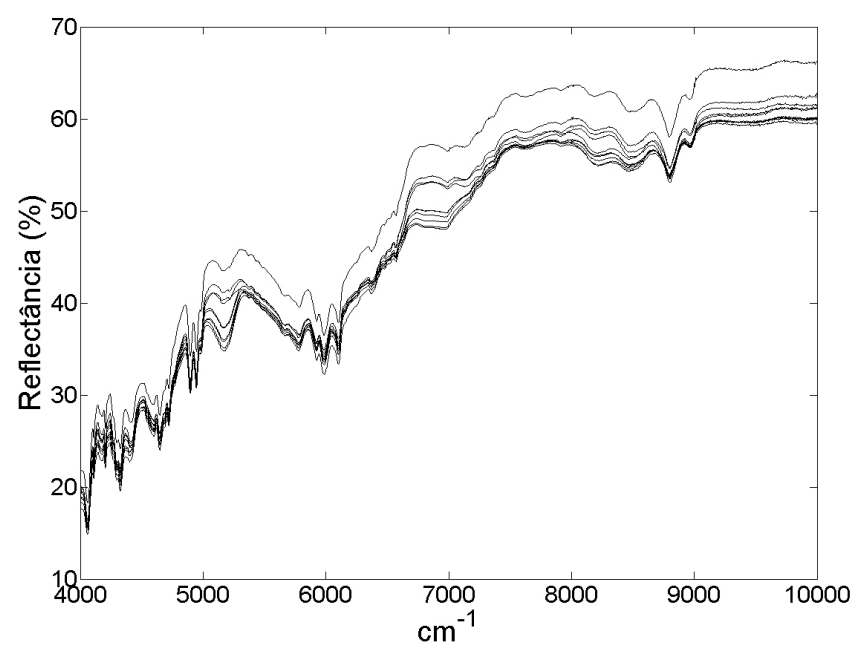

Figura 4S. Conjunto de espectros das amostras de validação em \% de reflectância 


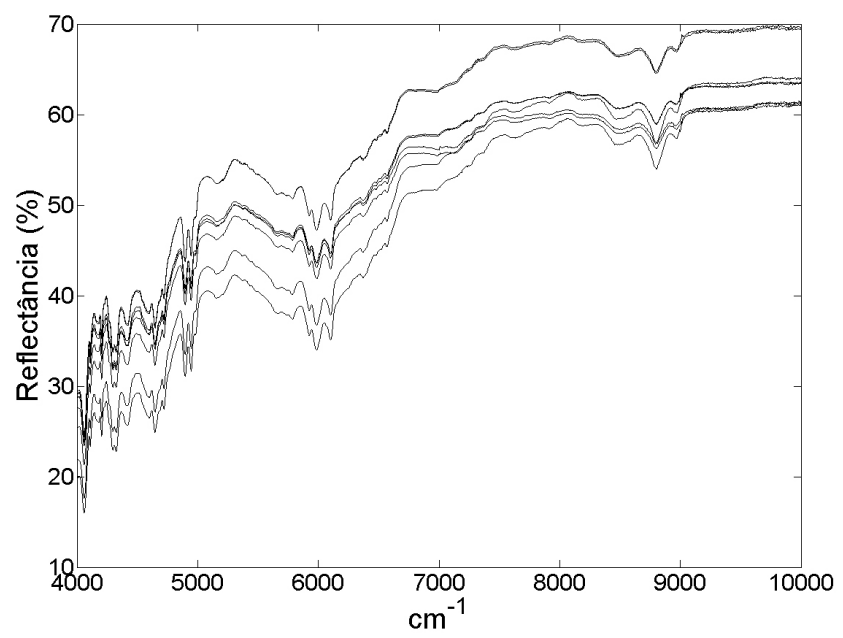

Figura 5S. Conjunto de espectros das amostras de medicamentos em \% de reflectância

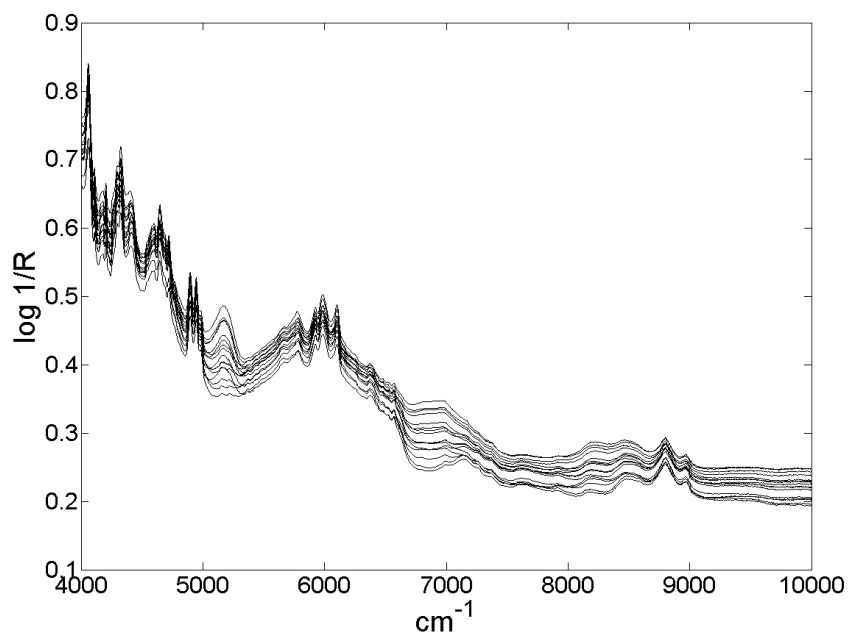

Figura 6S. Conjunto de espectros das amostras de calibração em log $1 / R$

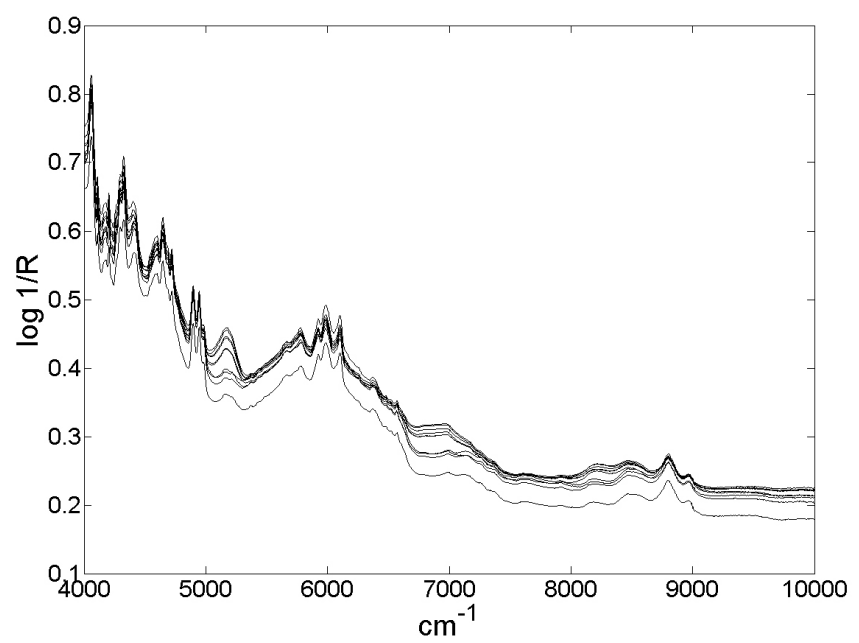

Figura 7S. Conjunto de espectros das amostras de validação em log $1 / R$

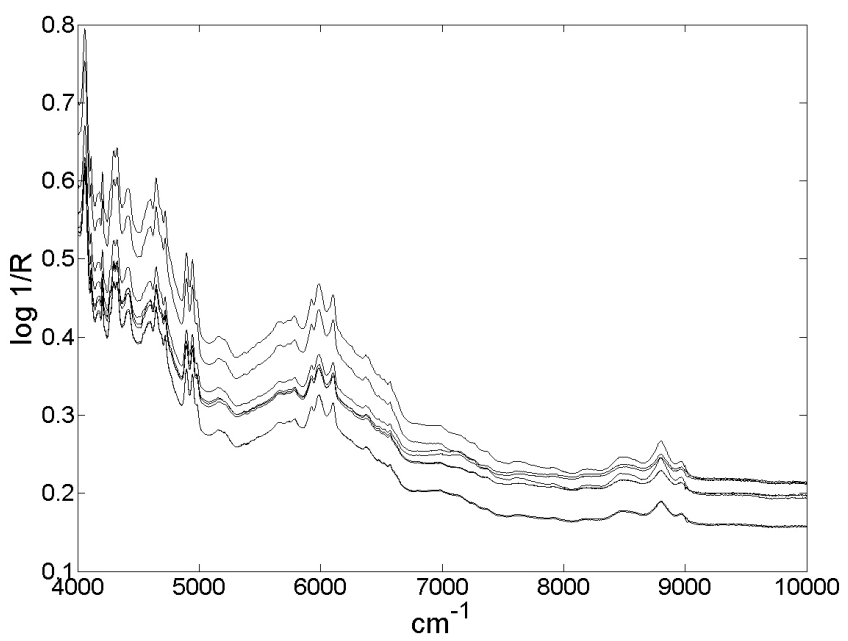

Figura 8S. Conjunto de espectros das amostras de medicamentos em log $1 / R$

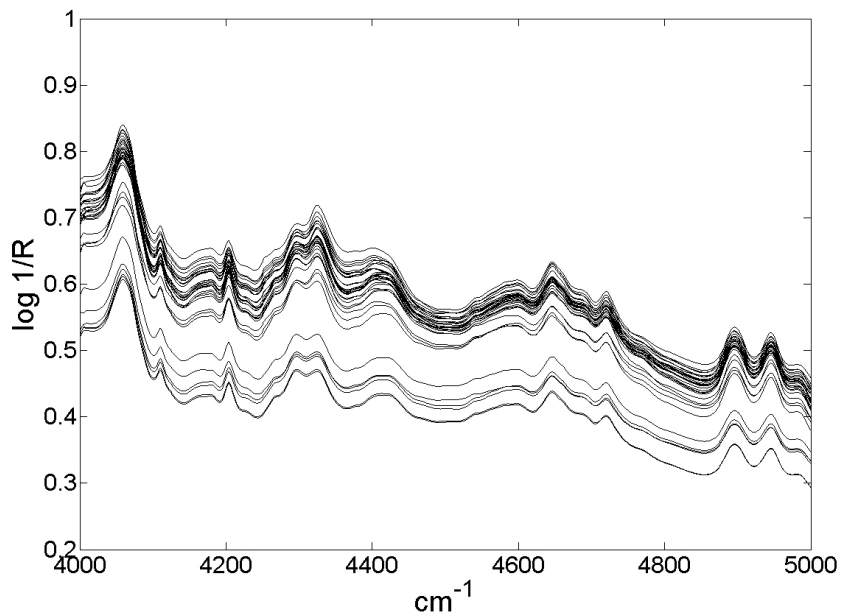

Figura 9S. Conjunto dos espectros após seleção da região espectral de interesse em $\log 1 / R$

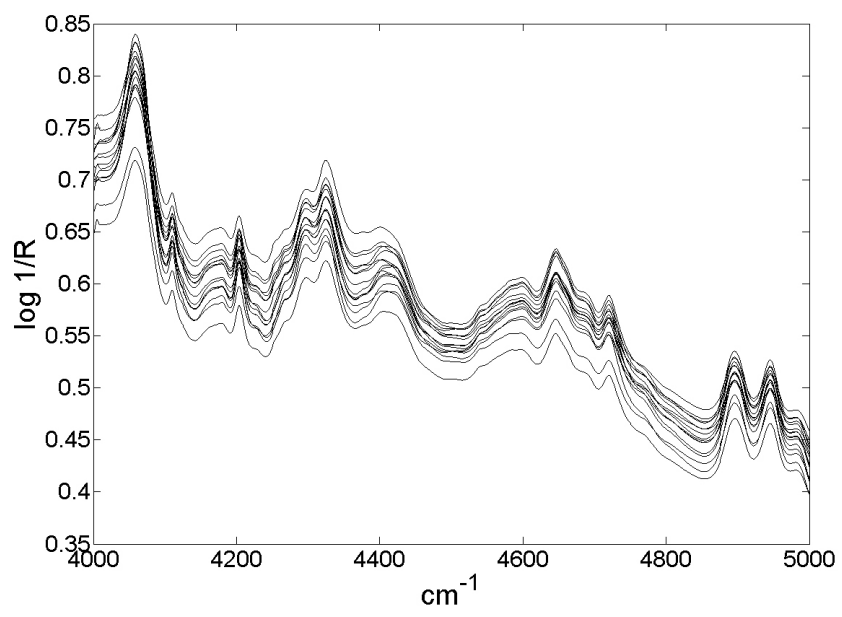

Figura 10S. Conjunto de espectros das amostras de calibração em log $1 / R$ após seleção da região espectral de interesse 


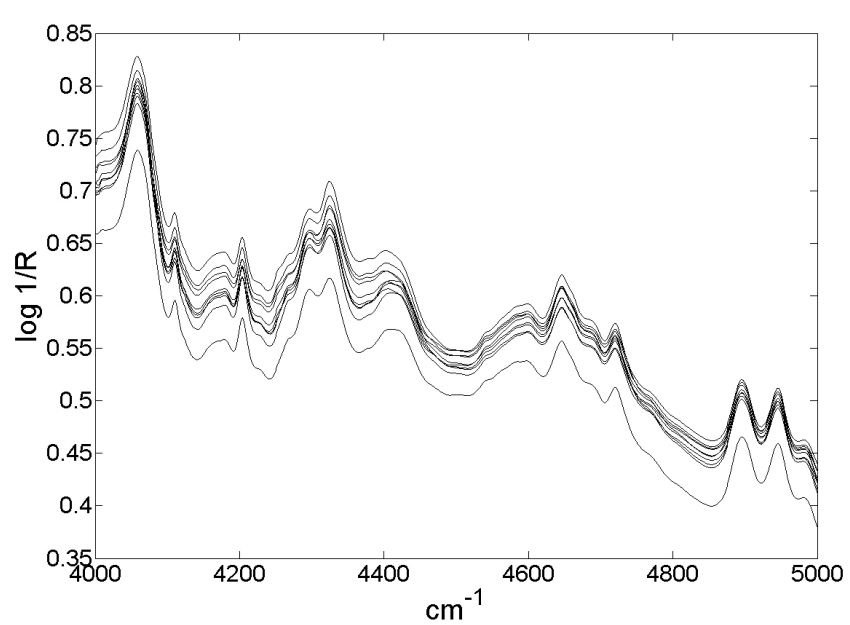

Figura 11S. Conjunto de espectros das amostras de validação em log $1 / R$ após seleção da região espectral de interesse

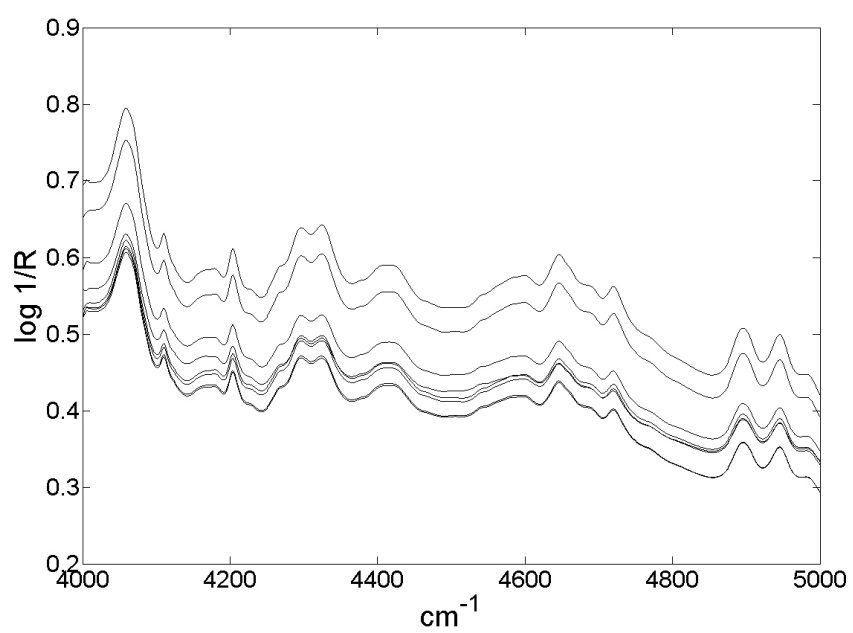

Figura 12S. Conjunto de espectros das amostras de medicamentos em log 1/R após seleção da região espectral de interesse

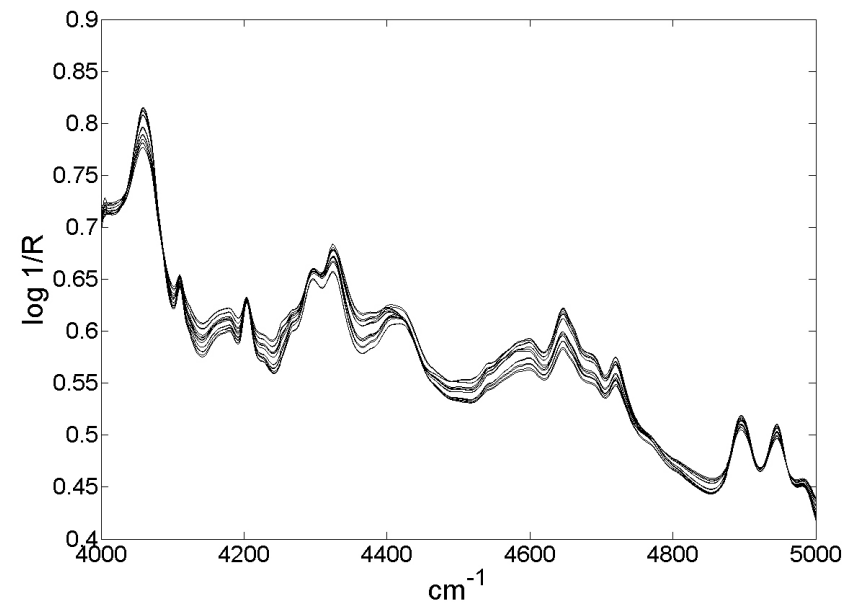

Figura 13S. Conjunto de espectros das amostras de calibração após correção por $M S C$

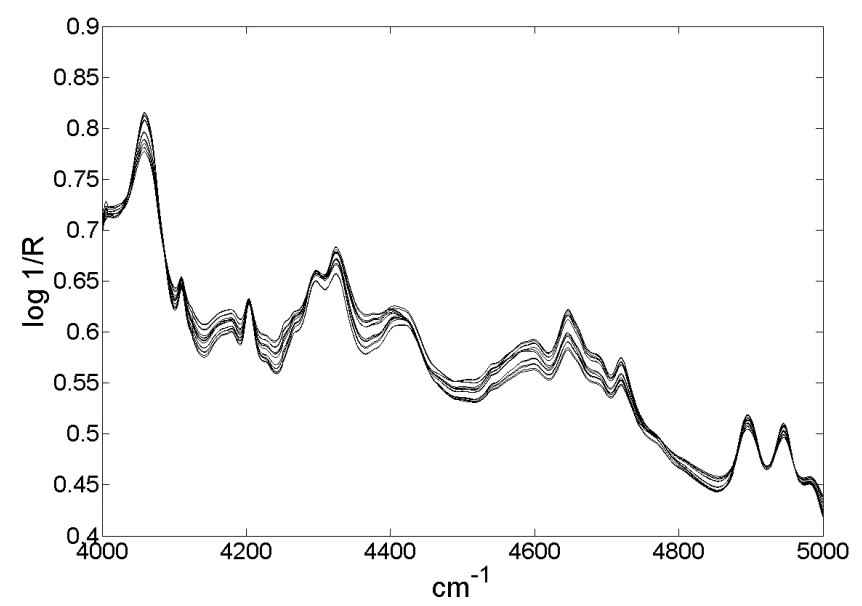

Figura 14S. Conjunto de espectros das amostras de validação após correção por MSC

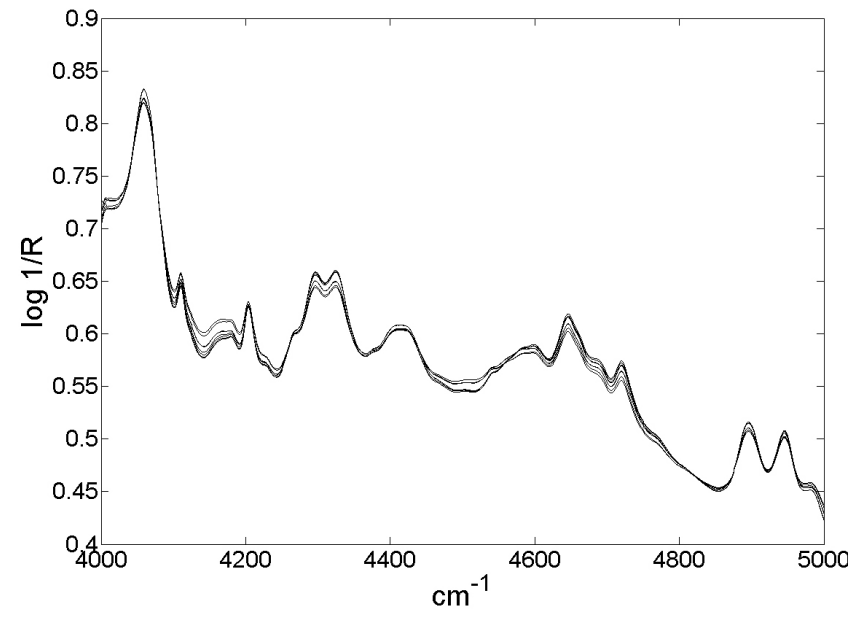

Figura 15S. Conjunto de espectros das amostras dos medicamentos após correção por MSC

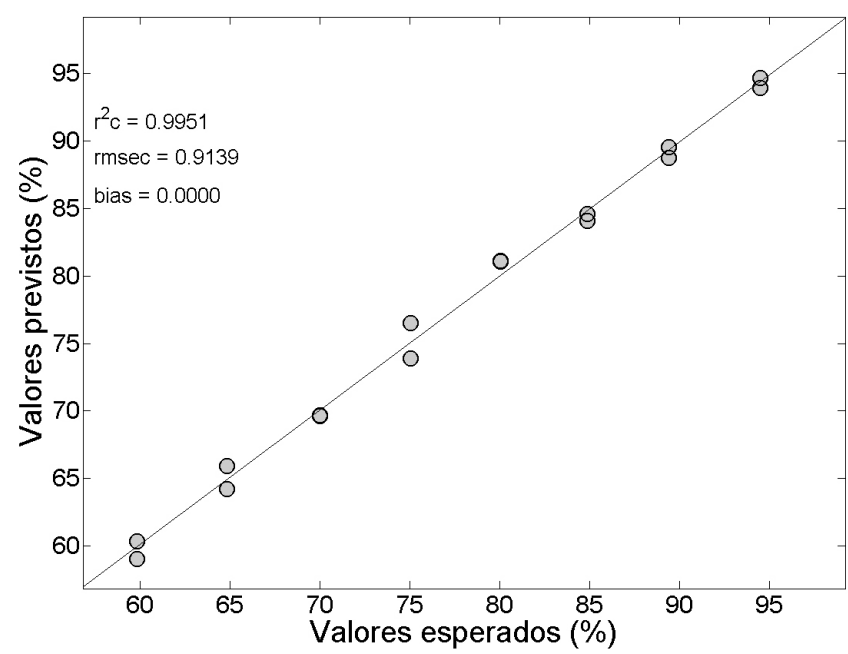

Figura 16S. Gráfico dos valores esperados vs valores previstos obtidos pelo modelo de calibração 


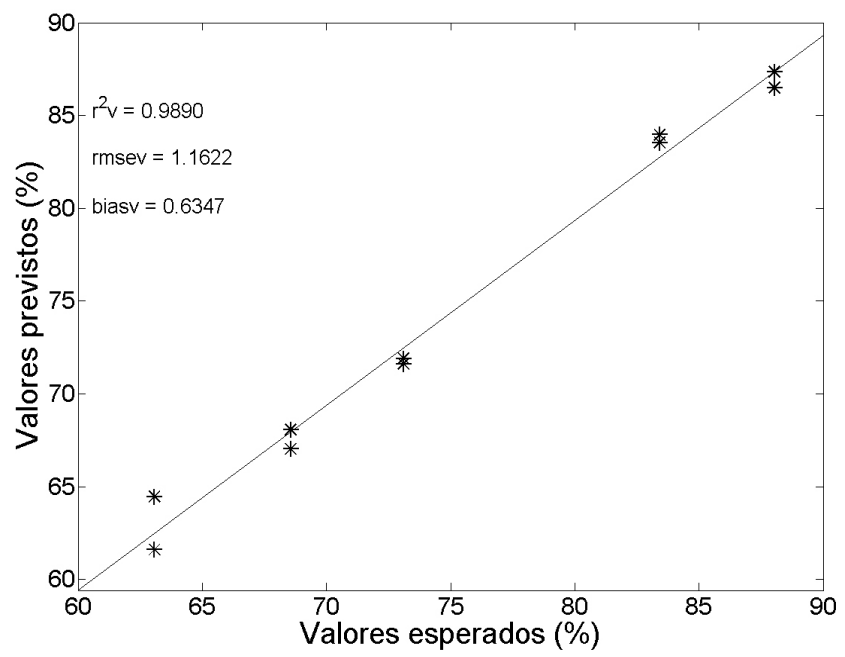

Figura 17S. Gráfico dos valores esperados vs valores previstos obtidos pelo modelo para validação externa 